\title{
12 (comunitania)
}




\title{
LA APLICALIÓN DEL PSICODRAMA PEDAGÓGICO A LA SUPERVIIIÓN EN TRABAJO SOCLAL' THE APICATTION OF PEDAGOGICAL PSYCHODRAMA TO THE SOCIAL WORK SUPEPVISION
}

\author{
Javier Arza Porras* \\ * Universidad Pública de Navarra (UPNA) \\ javier.arza@unavarra.es
}

\begin{abstract}
:
This article presents the basic principles of psychodrama and the main techniques of the called pedagogical psychodrama. We believe that the potential of his application in social work it hasn't been enough explored till now. The pedagogical psychodrama facilitates extraordinarily the decentration and the development of grand relevance competences like the flexibility, the spontaneity and the creativity. The article has been mainly write since the experience of the application of this approach to the practics in the social work degree. However, it argues that the pedagogical psychodrama it can be applied both in the educative supervision and in the profesional.
\end{abstract}

Key words: social work, supervision, university learning, practicum, pedagogical psychodrama.

\section{Resumen:}

En este artículo se presentan los principios básicos del psicodrama y las principales técnicas del denominado psicodrama pedagógico. Consideramos que las potencialidades de su aplicación a la supervisión en Trabajo Social no han sido suficientemente exploradas hasta el momento. El psicodrama pedagógico facilita extraordinariamente la descentración y el desarrollo de competencias de gran relevancia como la flexibilidad, la espontaneidad y la creatividad. El artículo ha sido escrito principalmente a partir de la experiencia de aplicación de este enfoque a las prácticas en el Grado de Trabajo Social. No obstante, se sostiene que el psicodrama pedagógico puede ser aplicado tanto en la supervisión educativa como en la profesional.

\footnotetext{
$1 \mathrm{Mi}$ agradecimiento a mis maestras Marian Becerro y Beatriz Basterretxea, del Instituto Garoa de psicodrama, y a mis compañeras de aprendizaje Ainara, Bittor, Isabel, Kris, Martina y Yolanda.
} 
Palabras clave: trabajo social, supervisión, formación universitaria, practicum, psicodrama pedagógico.

\section{Article info:}

Received: 22/12/2015 / Received in revised form: 02/06/2016

Accepted: 15/06/2016 / Published online: 01/07/2016

DOI: http://dx.doi.org/10.5944/comunitania.12.4

\section{Introducción: la estrecha vinculación entre supervisión y Trabajo Social}

Diversos autores y autoras (Hernández 1991; Fernández 1997; Miranda 2004; Puig 2011) señalan que el desarrollo de la supervisión ha sido paralelo a la evolución misma del Trabajo Social. Las organizaciones sociales filantrópicas que dieron origen al Trabajo Social en la transición entre el Siglo XIX y XX en Estados Unidos y Reino Unido ${ }^{2}$, ya utilizaban la supervisión. Las personas que ejercían labores supervisoras "normalmente eran colegas más experimentados que acompañaban al voluntario en sus demandas y sus necesidades de ayudarse a sí mismos para poder ayudar a los demás. Las precursoras del Trabajo Social profesional, Octavia Hill y Mary Richmond, asumieron funciones de supervisoras" (Puig 2011: 3).

Etimológicamente, supervisión deriva de las palabras latinas super (sobre) y videre (ver). En ese sentido, es una metodología que facilita a los y las participantes tomar distancia para poder ver desde arriba sus relaciones, sus acciones, sus posibilidades, sus preocupaciones, sus carencias o sus oportunidades. Es lo que Kersting (1999) denomina la perspectiva de la vista de pájaro, que permite observar y observarse con una mayor perspectiva y claridad. En nuestro contexto, la supervisión en Trabajo Social se ha desarrollado especialmente en el ámbito de la docencia universitaria, pero ha sido insuficientemente aplicada en el ejercicio profesional o en la formación continua.

En este artículo se presenta el marco teórico y metodológico general de la supervisión. Sin embargo, su principal valor añadido reside en que propone la incorporación del psicodrama pedagógico como parte imprescindible del modelo y de las técnicas de la supervisión en Trabajo Social. Aunque las propuestas del artículo pretenden ser extensibles a la supervisión profesional, los contenidos se refieren fundamentalmente a la supervisión educativa, ya que ha sido elaborado básicamente a partir de una experiencia de aplicación en las prácticas de Grado.

2 Como las Charity Organization Societies (COS). 


\section{La supervisión educativa}

Desde sus inicios, la formación universitaria de trabajadores y trabajadoras sociales ha contemplado las prácticas profesionales externas como una de sus señas de identidad. Los cambios experimentados en la última reforma universitaria, con el paso de Diplomatura a Grado, no han hecho más que reforzar esta característica, reafirmando que el practicum es uno de los ejes centrales de esta titulación.

El documento de Criterios para el diseño de planes de estudios de títulos de Grado en Trabajo Social recoge que las prácticas deben desarrollarse en centros y entidades desde las que se prestan servicios de Trabajo Social; y que deben incluir necesariamente dos elementos: la supervisión (en la Universidad) y la tutela profesional (en el propio centro de prácticas).

Una de las funciones esenciales de la supervisión educativa consiste en facilitar el proceso en el que el alumnado construye y adquiere un nuevo rol social: su rol profesional como trabajador o trabajadora social. Se trata de lograr que sea un proceso de construcción de la identidad profesional crítico y flexible, adaptado a una realidad social, política y profesional compleja y cambiante. Como señala Piaget (1983) el principal objetivo de la educación es crear personas capaces de hacer cosas nuevas, no simplemente repetir lo que otras generaciones han hecho. Asimismo, debe ser un proceso conectado con la propia identidad e historia del alumno o de la alumna, en el que pueda tomar conciencia y revisar sus valores, creencias o competencias, así como el resto de roles que conforman su yo. La autobservación es entonces otra de las áreas fundamentales que deben ser abordadas y entrenadas en la supervisión. Y para ello, una de las oportunidades fundamentales que ofrece esta metodología es la posibilidad de lograr la descentración, tanto de uno mismo o de una misma, como del sistema de intervención en el que se trabaja o se desarrollan las prácticas profesionales. Como decíamos anteriormente, la supervisión ofrece la posibilidad de observar y observarse a vista de pájaro.

\section{Marco teórico de la supervisión}

Los modelos teóricos en los que se inspira la supervisión son diversos y complementarios. En la literatura científica consultada (Hernández 1996; Ituarte 2012; Puig 2010; Berasaluze y Ariño 2014) podemos destacar cuatro grandes corrientes en las que se inspira esta metodología: teoría de los sistemas, teorías del aprendizaje y del conocimiento, teoría humanista y teoría constructivista. Sin embargo, y esta es una de las principales tesis de este artículo, no se contempla la enorme potencialidad que el paradigma del psicodrama puede aportar a la supervisión.

A continuación se resumirán las ideas esenciales de cada uno de los cuatro modelos clásicos de la supervisión. Con un mayor desarrollo, y en un apartado posterior, 
se plantearán las principales aportaciones del psicodrama pedagógico a la supervisión en Trabajo Social.

\section{Teoría de los sistemas}

De la teoría sistémica toma la clara conciencia respecto a la interdependencia entre los distintos elementos implicados en una situación. Asimismo, adopta la teoría de la comunicación humana de Watzlawick, Helmick y Jackson (1985) como paradigma de gran utilidad para comprender los procesos de retroalimentación que se producen en las interacciones humanas.

\section{Teorías del aprendizaje y del conocimiento}

Respecto a las teorías del aprendizaje, la supervisión entronca especialmente con los planteamientos de Kolb (1984), que destaca el papel que la experiencia juega en el proceso de aprendizaje. Este autor propone un ciclo del aprendizaje en el que se parte de una experiencia concreta, posteriormente se realiza una observación reflexiva sobre lo acontecido, luego se produce una conceptualización abstracta o conclusiones, para finalmente volver a probar en la práctica lo que hemos concluido.

En cuanto a las teorías del conocimiento, la supervisión conecta con las propuestas de Von Krogh, Ichijo y Nonaka (2001), que distinguen entre conocimiento tácito y explícito. El conocimiento tácito es aquel que forma parte de nuestro modelo mental, fruto de nuestra experiencia personal, e involucra factores intangibles como las creencias, valores, puntos de vista, intuiciones, sensaciones, etc. El conocimiento explícito, sin embargo, es aquel conocimiento que puede ser estructurado, sistematizado y transmitido fácilmente de un individuo a otro. La supervisión es una estrategia privilegiada para transformar conocimientos tácitos en explícitos.

\section{Teoría humanista}

Del enfoque humanista, representado principalmente por Rogers (1997), adopta el principio de autorrealización, por el que es imprescindible la participación de la persona supervisada en el análisis y la resolución de sus propias problemáticas. Asimismo, Rogers (1997) subraya la importancia del tipo de relación de ayuda que se establezca entre supervisor-a y supervisado-a, que debe estar basada en tres actitudes básicas: la empatía, la aceptación positiva incondicional y la autenticidad o congruencia. 


\section{Teoría Constructivista}

Del constructivismo, y especialmente de las aportaciones de Maturana y Varela (1984), toma la concepción de la realidad como una construcción mediada por el lenguaje. Las dificultades en los procesos de trabajo proceden entonces en gran parte de modelos de comunicación inadecuados. La supervisión debe permitir al sistema (grupo supervisado) visibilizar la diversidad de posibilidades de interpretación, procurando que pueda hallar interpretaciones más útiles. La aceptación de la intersubjetividad como elemento consustancial al ser humano y a su relación con el mundo, es parte fundamental de este modelo teórico.

\section{Marco teórico del psicodrama}

El psicodrama es un modelo que integra el cuerpo, las emociones y el pensamiento, ya que opta por una visión integral del ser humano. Apuesta con claridad por la acción, por el movimiento, por el hacer, aunque también tiene en cuenta la palabra en la imprescindible fase final de procesamiento grupal ${ }^{3}$.

A partir de varios autores y autoras (Bello 1999; Ramírez 1997; López y Población 1997; Schutzenberger 1975) se presentarán a continuación los conceptos básicos que Jacob Moreno, fundador de este modelo, aporta:

\section{Espontaneidad}

Moreno (citado por Ramírez 1997: 29) define la espontaneidad como "la respuesta nueva a una situación antigua o una respuesta adecuada a una situación nueva". Considera que es la clave para que el ser humano pueda encontrar respuestas adaptadas a las situaciones con las que permanentemente debe enfrentarse. De hecho, afirma que el psicodrama tiene como finalidad el desarrollo de la espontaneidad en el individuo, para que pueda de esta forma resolver sus problemáticas.

Para Ramírez (1997: 30) "la espontaneidad completa es la respuesta integral del ser humano completo: cuerpo, sentimientos, acciones, imaginación, pensamiento y determinaciones voluntarias". Como señala Moreno (citado por López y Población 1997) tiene que ser una respuesta plástica, flexible, que rompa o se libere de los condicionamientos que le llevan a respuestas repetitivas y desadaptadas. Moreno admiraba especialmente la espontaneidad de niños y niñas, que posteriormente era limitada por las rígidas estructuras sociales.

3 Describiremos esta fase en el apartado 6.2. 


\section{Creatividad}

Es un concepto estrechamente ligado al de espontaneidad. No se trata de crear "cosas" que nunca antes habían existido. Más bien se trata de encontrar relaciones nuevas entre elementos, explicaciones novedosas a fenómenos, maneras distintas de observar un acontecimiento,... y no tanto, o no únicamente, descubrir algo que no existía.

Moreno vincula la creatividad y la espontaneidad con el juego, y éste con el aprendizaje. Plantea que jugar y aprender tienen una raíz común en la infancia: el impulso de conocer, de explorar, de arriesgarse, de salir de los límites establecidos,... Por ello, insiste en incorporar el espíritu del juego en las escenas terapéuticas o pedagógicas, buscando con ello que aflore la espontaneidad y la creatividad.

\section{Catarsis o toma de conciencia}

A partir de la espontaneidad y la creatividad, el individuo puede llegar a la catarsis, a una "libertad de expresión que permite revelar las tendencias ignoradas, los móviles y las motivaciones ocultas, los viejos traumas, la movilización de lo que estaba fijado. Permite tomar conciencia y ser más accesible al cambio" (Schutzenberger 1977: 86). En el caso del psicodrama pedagógico hablaríamos más bien de toma de conciencia y no tanto de catarsis, que es un concepto más propio del enfoque terapéutico.

\section{Filosofía del momento}

Según Bello (1999: 27) para Moreno "todo instante vivido es un entrecruzamiento entre nuestra historia y la acción presente, que son inseparables". Toda la acción pedagógica o terapéutica se centra en el aquí y ahora para reconstruir la historia. Para Moreno el momento presente no es una parte de la historia, sino que la historia es una parte del momento presente, está en el presente.

Rol

Como señala Ramírez (1997: 34) Moreno define la personalidad por el conjunto de roles que desempeña el sujeto, y considera que "el psicodrama tiene por objeto ayudar a reconocer, formar, modificar y coordinar todos los roles o todas sus funciones". En ese contexto, el desarrollo de la espontaneidad y la creatividad se constituyen en una de las herramientas fundamentales para favorecer que el individuo sepa ofrecer respuestas flexibles y adaptadas a un contexto permanentemente variable, en el que deberá jugar un amplio repertorio de roles. López y Población (1997) emplean la 
metáfora del titiritero, que tiene en su baúl un montón de títeres. Siguiendo esa línea simbólica, podríamos decir que el mejor titiritero es el que sabe incorporar cada uno de esos personajes en el momento adecuado de la escena y que, incluso, va adquiriendo nuevos títeres para su baúl.

Partiendo de una visión integral del ser humano, Moreno considera que existen tres tipos de roles: roles psicosomáticos, ligados al cuerpo (por ejemplo, ingeridor); roles psicodramaticos, vinculados a la psique (por ejemplo, sumiso o rebelde); y roles sociales, relacionados con el contexto social (por ejemplo, madre o trabajador social). No obstante, no debemos ver los roles de manera aislada, sino que siempre se desarrollan en una escena dinámica y sistémica: los roles de un individuo interaccionan entre sí; pero a su vez se ponen en relación con los roles de otros individuos que participan en la escena, formando roles complementarios y/o contrarroles.

Tele

Es "la atracción o rechazo mutuo y a distancia entre dos o más personas, como el terapeuta y el paciente, o entre miembros del grupo" (Ramírez 1997: 37). La insistencia de Moreno en promover las relaciones grupales basadas en la libre elección, está basada en este concepto de tele. En las escenas psicodramáticas, Moreno promueve que el protagonista escoja las personas que han de representar los personajes de su drama. Moreno afirma que el protagonista intuye qué personas han experimentado situaciones semejantes a las suyas.

\section{Átomo social}

Moreno tenía una visión sumamente relacional del ser humano. Consideraba que, para desarrollar sus roles, cada persona precisa contar con otros individuos. En ese contexto surge el concepto de átomo social como "el conjunto de personas que son necesarias para el funcionamiento adecuado de un individuo en cada uno de sus roles" (Ramírez 1997: 40). Cada individuo tiene distintos átomos sociales dependiendo de los roles que desempeñe. Así, puede ser distinto el construido para el desarrollo de su rol profesional, que el configurado para su rol como padre. En cualquiera de ellos es fundamental, para que cumpla bien su función, que exista reciprocidad en el tele positivo que une a la persona con los componentes de su átomo social. Si no es así, su átomo social será endeble e ineficaz.

\section{Metodología y técnicas de la supervisión educativa}

Según Hawkins y Shohet (2000) en el espacio de supervisión coexisten dos sistemas interrelacionados: el sistema de intervención y el de supervisión. El sistema 
de intervención está compuesto por la persona supervisada, pero también por sus clientes y el contexto en el que trabaja, que están presentes aunque no sea físicamente. Por su parte, el sistema de supervisión está compuesto por el supervisor o la supervisora, el grupo supervisado y el contexto en el que se desarrollan las sesiones de supervisión. Siguiendo esta diferenciación, estos mismos autores distinguen un tipo de supervisión centrada en el sistema de intervención y otro que focaliza su atención en la dinámica interna del grupo de supervisión. Desde nuestro punto de vista, la diferenciación entre ambos sistemas es artificial, ya que el grupo de supervisión debe integrar todos estos elementos. Para ello, y siguiendo los postulados del psicodrama, el espacio de supervisión debe orientarse al aquí y ahora, pero considerando que en ese instante está integrado todo el sistema de intervención de cada miembro del grupo, y que todo lo movilizado en ese instante del sistema de supervisión regresará al contexto profesional de cada persona supervisada.

En todo proceso de supervisión educativa se desarrollan tres etapas:

\section{A. Fase preparatoria.}

Esta fase se orienta fundamentalmente hacia la preparación de la incorporación del alumnado a su centro de prácticas, por un lado, y hacia la constitución del grupo, por el otro.

\section{B. Fase de acompañamiento.}

En esta fase se produce la integración plena del alumnado en sus respectivos centros de prácticas. Es el momento de confrontar con la realidad todos los contenidos trabajados durante el Grado, pero también los miedos, las fantasías y las incertidumbres ante el proceso de prácticas, así como las competencias que el alumnado valoraba tener (o no tener). En esta interacción con la práctica se podrán releer, reforzar o reconducir todos los aspectos teóricos, técnicos o vivenciales que el alumnado traiga al grupo de supervisión.

\section{Fase de cierre y evaluación.}

La evaluación es continua, por lo que debe realizarse durante todo el proceso de supervisión. No obstante, en esta fase final se trata de asentar e integrar los aprendizajes adquiridos, desarrollando un proceso de evaluación a diferentes niveles: autoevaluación del propio alumno o alumna, evaluación del grupo de supervisión, evaluación del supervisor o supervisora y evaluación del centro de prácticas. En esta fase final también hay que cuidar especialmente la separación y el adecuado cierre de los procesos de vinculación profesional y personal que se han establecido en el centro de prácticas y en el grupo de supervisión. 
En cuanto a las técnicas, la supervisión es una metodología integrativa que emplea múltiples herramientas o técnicas. Berasaluze y Ariño (2014) repasan en su artículo las técnicas más presentes en la literatura especializada. Todas ellas son herederas de los modelos teóricos utilizados habitualmente en la supervisión. Por ejemplo, el contrato, que establece los compromisos y acuerdos básicos del grupo; las ruedas, tanto de toma de contacto o acogida como de cierre; la validación, que permite transmitir la aceptación incondicional defendida desde el modelo humanista; el feed-back, que aporta información a la persona sobre lo que transmite, hace o propone; la confrontación, que interpela a la persona para provocar o favorecer el cambio.

\section{Metodología y técnicas del psicodrama pedagógico}

El psicodrama suele ser vinculado únicamente a la terapia, pero su metodología y técnicas pueden ser empleadas también con una finalidad pedagógica, tal y como se trata de mostrar en este artículo. En el siguiente cuadro, Fernández (2009) plantea las diferencias entre ambos enfoques del psicodrama.

CUADRO 1.

Diferencias entre psicodrama pedagógico y terapéutico

\begin{tabular}{|c|c|c|}
\hline & Psicodrama Pedagóg ico & Psicodrama Terapéutico \\
\hline Finalidad & $\begin{array}{l}\text { Pedagógico y/o de formación } \\
\text { Efecto terapéutico secundario }\end{array}$ & Terapéutico \\
\hline Busca & $\begin{array}{c}\text { Cambio en un rol, de forma } \\
\text { flexible y espontánea } \\
\text { Aprendizaje o perfeccionamiento o } \\
\text { reaprendizaje de un rol }\end{array}$ & $\begin{array}{l}\text { Cambio estructural de los } \\
\text { sistemas-escenas internas }\end{array}$ \\
\hline Trabaja sobre & Escena manifiesta principalmente & $\begin{array}{l}\text { Escena manifiesta } \\
\text { Escena latente }\end{array}$ \\
\hline Utiliza & $\begin{array}{l}\text { Principalmente unas pocas } \\
\text { técnicas }\end{array}$ & Una amplia gama de técnicas \\
\hline $\begin{array}{l}\text { Fundamentos } \\
\text { teóricos }\end{array}$ & \multicolumn{2}{|c|}{ Los mismos } \\
\hline
\end{tabular}

Fuente: Fernández (2009).

\subsection{El role-playing pedagógico}

En el psicodrama pedagógico se utilizan prioritariamente cuatro técnicas: cambio de roles, doblaje, espejo y multiplicación dramática. Todas ellas son aplicadas dentro de una escena psicodramática, que en su modalidad pedagógica suele ser deno- 
minada role-playing. La escena puede responder a una situación real no resuelta, a una situación temida real o fantaseada, o al deseo de mejorar una competencia profesional relacional. La puesta en marcha de la escena permite el entrenamiento en las interacciones de roles entre seres humanos, en la comprensión de las diferentes visiones y posiciones. Además, al tratarse de una escena, podemos corregir, evaluar, repetir, reorientar, experimentar, nuestras respuestas.

Presentaremos a continuación las características de cada una de las cuatro técnicas mencionadas:

\section{Cambio de roles}

Es la técnica más característica del psicodrama. Su descripción más sencilla sería el dicho popular "ponte en mi lugar". Moreno se refería al cambio de roles como el verdadero encuentro con el otro. "Un encuentro dos a dos, ojo a ojo, cara a cara. $Y$ cuando estés muy cerca, yo quitaré tus ojos y los pondré en el lugar de los míos; y tú me quitarás mis ojos y los pondrás en el lugar de los tuyos; entonces yo te miraré con tus ojos y tú me mirarás con los míos" (Moreno, citado por Schutzenberger 1975: 87).

En la aplicación de esta técnica, se debe pedir a A que se ponga en el lugar de B, es decir, que escuche, hable, sienta,... desde la perspectiva cultural, profesional, social, del otro.Y lo mismo se debe solicitar a B respecto a A. Así, se desarrollará una nueva escena relacional en la que podrán surgir importantes aprendizajes sobre el rol del otro y el de uno mismo. A puede decir cosas a $B$ (que en realidad es uno mismo), pero desde una perspectiva nueva; $B$ puede experimentar qué se observa y qué se siente desde la perspectiva de $A$; ambos pueden encontrar nuevas respuestas al jugar desde posiciones diferentes.

Ejemplo 1. En una de las escenas representadas en supervisión, una alumna (A), que siempre se mostraba en el grupo muy tímida, retraída y apocada, representó una entrevista que desarrolló en su centro de prácticas, y en la que no se había sentido muy cómoda. Una vez representada la escena completa, se repitió un fragmento de la misma, pero incorporando un cambio de roles. La alumna $A$ pasó a asumir el rol de $B$, que representaba a una usuaria muy enfadada, y viceversa. A se metió muy bien en el rol de $\mathrm{B}$ e impresionó al grupo por la fuerza y energía que transmitía. Realmente parecía otra persona. En el procesamiento grupal posterior, la alumna manifestó haberse sentido muy bien al poder expresarse tan enfadada y enérgica. Transmitió también que lo que observó en la alumna que representaba su rol (retraimiento, timidez), hacía que se enfadase aún más. En sesiones grupales e individuales posteriores, esta alumna pudo seguir trabajando en torno a lo que generaba en las demás personas su rol de apocada, las consecuencias positivas y negativas que tenía para ella, así como lo que podía representar en su nuevo rol profesional. 


\section{Doblaje}

En esta técnica otra persona del grupo, o la propia persona supervisora, poniendo las manos en los hombros del sujeto, debe tratar de expresar lo que percibe que no manifiesta. Se emplea cuando se observa que el sujeto tiene muchas dificultades para expresar (verbal y/o corporalmente) sus contenidos internos.

Ejemplo 2. Una alumna, que realizaba sus prácticas en una asociación que trabaja con personas inmigrantes, plantea que se siente muy insegura en las entrevistas de acogida. En la escenificación de la entrevista, la alumna fue planteando una larga lista de preguntas a modo casi de cuestionario. La alumna que representaba a la persona entrevistada iba contestándolas con frases cortas y expresión aburrida. Otra alumna del grupo se situó espontáneamente detrás de la entrevistada e hizo un doblaje diciendo "pero señorita, todo eso que me pregunta me lo han preguntado ya en mil sitios ¿para qué lo necesita saber? ¿qué me ofrecen en este sitio?". Este doblaje reorientó la dinámica de la escena. Posteriormente, en su centro de prácticas, la alumna propuso replantear el guión y el estilo de entrevistas de acogida que se realizaban.

\section{Espejo}

Se trata de imitar (no caricaturizar) la manera en la que una persona se expresa verbal y no verbalmente. De esta manera, el sujeto puede observarse desde fuera como en un espejo.

Ejemplo 3. Un alumno propone escenificar una entrevista familiar que fue realizada en su centro de prácticas y que, desde su punto de vista, salió mal. En el transcurso de la representación, el supervisor propone hacer una parada y hace de espejo del alumno en la repetición de un fragmento de la escena. En ese fragmento de la entrevista, una de las personas de la familia acusa a su padre de haber abusado de ella en el pasado. El alumno, en su rol de entrevistador, responde con un lacónico “bueno, y por lo demás, ¿cómo te encuentras?". La aplicación de esta técnica permite que el alumno se observe desde fuera e identifique algunos errores verbales y no verbales, además de facilitar que sea consciente de los bloqueos que le generan las situaciones de abuso intrafamiliar y de cómo tiende a reaccionar ante ellas.

\section{Multiplicación dramática}

Es la técnica más característica y específica del psicodrama pedagógico. Supone que cada miembro del grupo actúe como el protagonista, pero desde su propio estilo y criterio. En ese sentido, no se trata de un doblaje ni de un espejo. De esta forma se muestra la multiplicidad de alternativas existentes ante una misma situación, evi- 
tando respuestas fijas y estereotipadas. La persona que presentó la escena puede llevarse muchos modelos para poder construir su propia respuesta adaptada.

Ejemplo 4. Una alumna expresa una gran inquietud en la relación con su tutor de prácticas. Lleva dos semanas en el centro y no se siente acompañada por él. Varias compañeras del grupo le plantean a la alumna que hable con él. La alumna tiene miedo a hacerlo. No sabe cómo se lo tomará y si será contraproducente para su nota final. El supervisor propone representar una escena en la que la alumna tiene una reunión con su tutor de prácticas para exponer cómo se siente. En la escena, de manera sucesiva, varias personas del grupo van representando cómo actuarían en el lugar de la alumna.

\subsection{Fases en la aplicación de las técnicas psicodramáticas}

\section{Introducción y caldeamiento}

Esta es una fase de gran relevancia en el psicodrama. El grupo debe pasar, del contexto social de su cotidianeidad, al aquí y ahora del contexto grupal. Cada persona debe tomar conciencia de su cuerpo, de su mente y de sus emociones, para prepararse a la interacción con otros roles propios y ajenos, y a la posibilidad del cambio espontáneo y creativo.

Dependiendo del momento del grupo, y del tipo de técnica o escena a representar, el caldeamiento grupal puede tener mayor o menor intensidad. En el caso de la supervisión educativa en el contexto universitario, en las primeras sesiones el caldeamiento puede ser de tipo verbal ${ }^{4}$, pero posteriormente se pueden incorporar otras técnicas más activas que impliquen la activación física ${ }^{5}$ o emocional ${ }^{6}$.

El caldeamiento grupal también tiene como objeto el surgimiento de la escena a representar. En ocasiones, el propio caldeamiento permite que emerja con claridad una propuesta. Si no es así, otra posibilidad puede ser el plantear en subgrupos la puesta en común de hipotéticas escenas a ser representadas. Cada subgrupo debe elegir una de ellas. Posteriormente se hace una puesta en común y se elije la que más interese al grupo. Alguna de las no elegidas puede ser reservada para otra sesión.

\footnotetext{
4 Por ejemplo, a través de rondas para poner en común experiencias, estados de ánimo, resistencias ("a mí no me gustan este tipo de dinámicas", "esto son tonterías",...) y a través de explicaciones del supervisor o la supervisora sobre el sentido de este tipo de técnicas.

5 Por ejemplo, paseos por la sala realizando diferentes movimientos guiados por el supervisor o la supervisora.

6 Por ejemplo, visualizaciones guiadas por el supervisor o la supervisora. Posteriormente se explicará esta técnica.
} 


\section{Caldeamiento específico}

En esta fase, y una vez elegida la escena a representar, la persona supervisora debe apoyar al o la protagonista en las tareas de preparación de la escena. Para ello, debe ayudarle a definir el escenario, situando al público en el espacio más adecuado y colocando los objetos que faciliten la representación (sillas, mesas, etc.). Posteriormente tiene que invitarle a elegir al resto de personas que van a participar en la representación (por ejemplo, una persona usuaria, un o una profesional, etc.). También debe proponerle que ponga en situación a estas personas, ayudando así a que se metan en el rol a través de una explicación de sus principales características. No obstante, también se propone que, a partir de esas explicaciones, cada persona pueda usar la espontaneidad y la creatividad en la representación de su rol.

\section{Dramatización}

Antes de la dramatización se debe insistir, tanto a las personas que representan roles como a las que hacen de público, la máxima concentración en sus respectivos papeles.

Durante la dramatización, la persona supervisora debe ir valorando la incorporación de las diferentes técnicas que hemos analizado. Por lo general, lo idóneo es que se pueda representar la escena completa (con la posibilidad de incorporar algún doblaje) y posteriormente se pueden repetir fragmentos incorporando el cambio de roles, el espejo y la multiplicación dramática.

\section{Procesamiento}

En el procesamiento, también llamado eco grupal, se abandona la escena y el grupo vuelve a situarse en círculo. La persona supervisora propone que, quienes han jugado algún rol, puedan expresar cómo se han sentido desde el mismo. Posteriormente amplía la puesta en común a las personas que han participado como público. En una segunda ronda, propone que extraigan conclusiones de la escena que puedan servir para la construcción de su identidad profesional. Finalmente, se invita al o a la protagonista a que haga una valoración sobre lo que la escena y el grupo le ha aportado.

En ocasiones, el procesamiento puede suponer un caldeamiento para continuar con la escena o para representar una escena nueva. No obstante, el formato de las sesiones de supervisión en el contexto universitario (habitualmente de dos horas de duración), dificulta que esto sea posible en una misma sesión. 


\subsection{Otras técnicas del psicodrama pedagógico}

En este apartado proponemos otras técnicas que se pueden utilizar al margen de las cuatro técnicas básicas del role-playing. Pueden ser aplicadas en el contexto de un caldeamiento grupal, pero también pueden tener sentido como técnica principal de una sesión. En cualquier caso, las fases para su aplicación son las mismas ya referidas en el apartado anterior.

\section{Escultura}

Es una técnica de expresión simbólica. El sujeto expresa con una posición congelada de su cuerpo lo que considera la esencia de una escena, ya sea presente, pasada o imaginada. El supervisor o la supervisora debe animar a que la escultura sea representada sobre todo desde la espontaneidad y no tanto desde la racionalidad. Una vez realizada, y sin salir de la misma, cada persona va expresando en voz alta las emociones que siente desde la escultura. También puede poner un título a la misma. Por las características de esta técnica, es recomendable que previamente se plantee alguna actividad de caldeamiento.

Ejemplo 5. En la Fase de Introducción de algunos grupos de supervisión hemos utilizado la técnica de la escultura para abordar las experiencias del alumnado en periodos previos de prácticas. En primer lugar se solicita al alumnado que piense en escenas significativas de sus prácticas; posteriormente se les solicita que individualmente elijan la más significativa y la representen con una escultura; en la fase final de procesamiento de esta técnica aflora información de gran utilidad para el diagnóstico individual y grupal.

\section{Visualización}

A través de la visualización se pretende que el sujeto construya mentalmente una escena. Es preciso que se realice previamente un caldeamiento, para lo que se invita a que cada persona cierre los ojos e inicie un proceso de concentración. El supervisor o la supervisora irá guiando al grupo en un recorrido mental por su trayectoria vital, o por una parte específica de la misma. Dependiendo del objetivo de la visualización, invitará a que cada persona se detenga en determinados momentos y observe distintos aspectos de sus experiencias y vivencias. También se le puede invitar a que introduzca personajes nuevos o a que se proyecte hacia el futuro.

Ejemplo 6. En una sesión, en la que trabajamos el acompañamiento social, invitamos al alumnado a visualizar el encuentro con alguna persona que les hubiera acompañado de manera eficaz durante su trayectoria. En ese encuentro debían conversar mentalmente con ella sobre cómo fue ese acompañamiento, qué hizo esa per- 
sona para que el acompañamiento fuese eficaz, etc. Posteriormente debían encontrarse con otra persona a la que ellos o ellas hubiesen acompañado con eficacia. También debían conversar con ella sobre cómo fue la experiencia.

\section{Representación del átomo social}

Se trata de representar, de manera gráfica, la red de relaciones personales que son significativas para el desarrollo de un rol. Para representarlo, en una hoja o cartulina cada persona dibuja un primer círculo en el que escribe su nombre. Posteriormente se dibujan otros círculos concéntricos. En ellos, el sujeto debe ir anotando el nombre y apellidos de cada una de las personas que forman parte de su átomo social. Según el grado de proximidad con el sujeto, los nombres serán escritos en un círculo más o menos cercano al núcleo. Una vez realizado, cada persona debe reflexionar sobre lo que le transmite la observación de su átomo social: qué le sorprende, qué le gusta, qué le falta, qué le aporta cada persona que forma parte del átomo social, etc. En algunos casos, la realización de esta dinámica puede servir como caldeamiento del que surja una escena en la que algún miembro del grupo dialogue con su átomo social, o con una parte del mismo.

Ejemplo 7. En supervisión invitamos a que el alumnado realice un átomo social centrado en su desarrollo profesional, situando a las personas que son significativas para la construcción de su identidad como profesionales delTrabajo Social. Una vez realizado y analizado, les invitamos también a que puedan tener, fuera del grupo de supervisión, conversaciones de aprendizaje con las personas que consideren más significativas de ese átomo social. Esta técnica puede ser aplicada al inicio y al final del proceso de supervisión, para poder evaluar de esta manera la evolución de la persona. También puede ser realizada con un enfoque prospectivo: ¿cómo me gustaría que fuese mi átomo social en el futuro?

\section{Conclusiones}

La supervisión ha estado presente en el Trabajo Social desde los inicios de nuestra disciplina. Actualmente, su presencia en la formación universitaria es unánime, pero no ha logrado consolidarse en el ámbito profesional como metodología de mejora de la intervención y de desarrollo profesional.

La supervisión es un método que integra múltiples enfoques y modelos en su desarrollo. Sin embargo, hasta el momento no ha contemplado suficientemente las aportaciones teóricas, metodológicas y técnicas del psicodrama pedagógico. La construcción-reconstrucción de la identidad profesional, que es uno de los objetivos fundamentales de los procesos de supervisión, puede ser abordada de una manera muy eficaz a través de la metodología y las técnicas propuestas desde el psicodra- 
ma pedagógico. Técnicas como el cambio de roles, el espejo, la escultura o el átomo social, facilitan procesos de enseñanza-aprendizaje caracterizados por la flexibilidad y el cambio adaptativo. Además, refuerzan competencias profesionales como la espontaneidad o la creatividad, de gran valor para contextos complejos y cambiantes como los actuales.

Finalmente, subrayar que la aplicación del psicodrama pedagógico, pero también de otras metodologías y técnicas de la supervisión, exige una formación específica y continuada por parte de aquellos y aquellas profesionales que deseen ejercer una labor supervisora. Además, es fundamental que se mantenga el compromiso de incorporación de la supervisión como eje fundamental del practicum en el Grado de Trabajo Social, pero también que ese compromiso se extienda a la instauración de la supervisión como una herramienta imprescindible en el ejercicio profesional.

\section{Bibliografía}

Bello, M.C. 1999. Introducción al psicodrama. Guía para leer a Moreno. México D.F: Colibrí.

Berasaluze, A. y Ariño, M. 2014. De la supervisión educativa a la profesional. Cuadernos de Trabajo Social. Vol. 27. №.1. https://revistas.ucm.es/index.php/CUTS/article/view/42464

Fernández, A. 2009. "Métodos adjuntos, integraciones y derivaciones del psicodrama". En Manual de formación de la asociación española de psicodrama. Madrid: Asociación Española de Psicodrama.

Fernández, J. 1997. La supervisión en el trabajo social. Barcelona: Paidós.

Hawkins, P. y Shohet, R. 2000. Supervision in the helping professions. Buckingham-Philadelphia: Open University Press.

Hernández, J. 1991. Acción comunicativa e intervención social. Madrid: Popular.

Hernández, J. 1996. La supervisión como modelo didáctico en la formación del prácticum en los nuevos planes de estudio. En C. Lobato (ed.), "Desarrollo profesional y prácticas/prácticum en la universidad". Bilbao: Servicio Editorial UPV/EHU.

Kersting, H. J. 1999. La supervisión como sistema de reflexión de la praxis profesional: paradojas y oportunidades. En J. Hernández (comp.), "La supervisión: Calidad de los servicios. Una oportunidad para los profesionales de ayuda". Pamplona: Eunate.

Kolb, D., 1984. Experiential Learning: experience as the source of learning and development. Englewood Cliffs, New Jersey: Prentice-Hall.

López, E. y Población, P. 2000. Introducción al role-playing pedagógico. Bilbao: Desclée de Brouwer.

Maturana, H. y Varela, F. 1984. El árbol del conocimiento. Santiago de Chile: Editorial Universitaria.

Miranda, M. (coord.) 2007. Criterios para el diseño de planes de estudios de títulos de Grado en Trabajo Social. Madrid: Conferencia de Directores/as de Centros y Departamentos de Trabajo Social.

Ovejas, C. et al. 2012. Encuesta sobre prácticas externas y Trabajo Fin de Grado en Trabajo Social. Revista Alternativas. №. 19. http://rua.ua.es/dspace/handle/10045/26722 
Piaget, J. 1983. El criterio moral en el niño. Barcelona: Editorial Fontanella.

Puig, C. 2011. La supervisión en los equipos de Servicios Sociales: una oportunidad para la reflexión, el pensamiento y el cuidado de los profesionales. Perspectivas Sociales. Vol. 13. $\mathrm{N}^{\circ}$. 1. http://dialnet.unirioja.es/servlet/articulo?codigo $=3705673$

Ramírez, J.A. 1997. Psicodrama: teoría y práctica. Bilbao: Desclée de Brouwer.

Rogers, C. 1997. Psicoterapia centrada en el cliente. Barcelona: Ediciones Paidós Ibérica.

Schutzenberger, A. 1975. Introducción al role-playing. Madrid: Marova.

Von Krogh, G., Ichijo, K. y Nonaka, I. 2001. Facilitar la creación de conocimiento. Cómo desentrañar el misterio del conocimiento tácito y liberar el poder de la innovación. México: Oxford University Press.

Watzlawick, P., Helmick, J. y Jackson, D. 1985. Teoría de la comunicación humana. Interacciones, patologías y paradojas. Barcelona: Editorial Herder. 


\section{ARTICULOS/ARTICLES}

Mediación, ciencia social emergente / Mediation, emerging social science

Francisco Javier Gorjón Gómez

La red de apoyo en mujeres con distintos grados de maltrato doméstico en Ciudad

Juárez, México / The social support network among women in different conditions

of intimate partner violence from Ciudad Juárez, México

Rogelio Hernández Rodríguez.

Tendencias del Trabajo Social en el ámbito de los Servicios Sociales desde la

perspectiva profesional. Estudio Delphi / Trends of Social Work in the field of socia

services from the professional perspective. Delphi study

Arantxa Hernández-Echegaray.

La aplicación del psicodrama pedagógico a la supervisión en Trabajo Social /

The aplicattion of pedagogical psychodrama to the Social Work supervision

Javier Arza Porras

Crisis y descontento. El aumento de la abstención crítica en el sur de Europa, 2002-2012

/ Crisis and dissatisfacion. The rise of critical abstention in southern Europe, 2002-2012

Rocío Alarcón Raser...

Empatía y habilidades sociales en enfermeras asistenciales en el Servicio Madrileño

de Salud / A study of empathy and social skills in registered nurses in Madrid Health Service

Diego Ayuso Murillo.

\section{RESEÑAS/REVIEWS}

Francisco Gorjón Gómez y Jorge Pesqueira (coords.). La ciencia de la mediación /

Science of mediation 2015

(por Cristina Mariana Lizaola Pinales)

Págs 127-129 